\title{
3D Motion Capturing Xography for Hand Trailing and Signal Appreciation of Video Games
}

\author{
P. S. Jagadeesh Kumar \\ Department of CSE \\ KL University, Vaddeswaram \\ Guntur, Andhra Pradesh, India
}

\author{
Yanmin Yuan \\ Research Scientist \\ Zheing Bell Lab \\ Chai Chee, Singapore
}

\author{
Mingmin Pan \\ Intel Research Lab \\ Yuhangtang Road \\ Hangzhou, China
}

\begin{abstract}
The asymmetrical subterfuge anthropomorphizes a simmering 3D motion of hand that is espoused to a predictable outline which classifies the Xography for signal appreciation of video games. The hand can be trademarked into numerous signal brochures or in uncommon movement trails. This gearshift the evolution of video games built on Xography for hand trailing and signal appreciation method. Essentially, the determination of this manuscript is to eloquent an energetic and reasonable user-input design to make accessible a hand trailing method with the goal that other scientists may inflate inventive claims of such popper. This practice is initially persuaded on two decisive hoboes; hand trailing and signal appreciation from 3D motion capturing Xography. Towards deliberate movement that enunciates from hand, Xography technique protonate on pointer speed restoration.
\end{abstract}

\section{General Terms}

Bayes Rule, Kronecker Delta Utility, Gaussian Distribution, Covariance Matrix, Kinematic Movement

\section{Keywords}

Xography, Electronic Computer Game, Hand Topography, Pointer Trailing, Signal Appreciation.

\section{INTRODUCTION}

Xography was curved by Whatmore and Rothstein towards caring their equipment with an exceptional and attractive trade name. The idiom is a tricky one to outline all the way through both current and precedent picturesque records in addition to magazines. The name materializes often throughout the years the Xograph was been fashioned, habitually in the 1960 s to the mid1970s, but then started to vanish from lenticular substance and magazines. The Xograph was employed in many dissimilar outlines, together with postcards and sports cards. [1] The idiom "Super Xograph" later came out with lenticular baseball cards and postcards in addition, but is not known in any chronological, methodical, and other magazines. Technological and photogenic lexicons and handbooks from the interlude afford a perceptive of the reputation of the expertise from the 1960s. The 1973 Focal Dictionary of Photographic Technology delineates Xography, panoramagrams, lenticular descriptions, and a few major vocabularies essential for the appreciation of how these photographs been fashioned.

Beginner's illustration and alphabetical tidy formulate the Focal Dictionary of Photographic Technologies easy to find the method and a vital allusion when considering associated patents. The existence of parallax panoramagram techniques throughout its fabrication reveals its importance and viable assurance surrounded by the photographic diligence. Xograph is located within the superior umbrella of stereo photography, and furthermore as a pioneer to holography. The Xograph is a photomechanical entity, produced by means of the procedure identified as offset lithography, though according to Rothstein, the company expected to progress towards the rotogravure printing techniques [2]. Variable warmth and moisture during the creation cause development and retrenchment of the synthetic coating, which could affect in misalignment of the lenticular partition and a deformation of the image stratum, generates a ruinous entity. Even though this setback was palpable instantaneously, also other perpetuation concern thus exists.

\section{3D MOTION CAPTURING XOGRAPHY}

Xographs were conventional to the society on 25 February 1964 by LOOK magazine, a periodical bent by Cowles Communications Limited. The Xographs viably fashioned photomechanical possessions; generate the delusion of threedimensional space in flamboyant color on a synthetic substrate with a linear textured facade. As the objects, do not necessitate the employ of ocular screening contrivance, it is classified as autostereoscopic. As this was the preliminary autostereoscopic photograph easily reached for those to acquire and amass these substances obligatory to be revisited. Researchers of the journal limiting Xographs hypothetical to be exceptional to determine more information on these threedimensional substances, nevertheless, very little exists. The aspiration to build a three-dimensional effect on a twodimensional facade was apparent in the actual precipitate times of cinematography [2,3]. Stereographic daguerreotypes were created and out looked through a stereoscope. Threedimensionality was endeavored and tested with from the mid of eighteen hundred throughout numerous techniques and methods, principal to the Xograph. This arrangement of threedimensional photogenic process is significant to comprehend how the Xograph get nearer to survive. To construct a photograph that requisite no extra screening equipment to create the delusion of the third facet, and suggestion to this method as pseudoscopic rather than stereoscopic, connotation it formed the delusion of the third dimension devoid of the inexorableness of optical gadgets like that enforced for stereographs [4]. The parallax stereogram needed an in-screen camera, a lot like the afterward parallax panoramagram. Lenticular monitor was positioned cautiously at the front of a sensitized plate but not including corporeal contact surrounded by the camera.

To understand the preferred three-dimensional outcome, the parallax stereogram desires an impeccable coalition with the viewer's sightline, and had to be inspected at a conserved distance. When the parallax stereogram was practiced, it was written as a buoyant and then positioned onto a goblet sustain. A glass partition was added to the exterior with obscure perpendicular lines that flawlessly allied with those on the glass photograph underneath. When detained at the best 
distance, which according to Ives, was just about the focal length of the lens, the viewer's right and left eyes would observe the upright wreckage of the image fashioned by its correlating orifice within the camera. Consequently, the right eye would perceive only the fraction of the photograph created by the right orifice, and the left eye would only perceive the fraction of the photograph created by the left orifice. With this ideal coalition and screening aloofness, the three-dimensional effect was attained with the naked eye. In this manuscript, the dialect "3D Motion Capturing Xography" refers to the procedure of customized Xograph scheme with the most modern three-dimensional motion capture process exclusively intended for computer game fabrication supported on pointer trailing and signal appreciation.

\section{3D MOTION ACQUISITION AND VIDEO GAMES}

Xography put forward the modus operandi of tailored Xograph proposition with 3D motion capture carried out absolutely deliberated for video game production sustained on pointer trailing and signal appreciation. Specified the interpretations up to time $t, Z_{1: t}$, the aspiration is to guesstimate the subsequent allotment of the state $x_{t}$ which includes the joint angles and 3D position of the hand. With the Markov hypothesis, the surveillance at time $t$ is independent of all precedent interpretations in specified $x_{t}$, the consequent is rationalized by means of the Bayes rule when accomplishing the surveillance at time $t$ :

$p\left(x_{t} \mid z_{1: t}\right)=c_{t} p\left(z_{t} \mid x_{t}\right) p\left(x_{t} \mid z_{1: t-1}\right)$

where $c_{t}$ is a normalization invariable and $p\left(z_{t} \mid x_{t}\right)$ and $p\left(x_{t} \mid z_{1: t-1}\right)$ are probability and preceding distribution, correspondingly. The preceding is figured as pursues:

$p\left(x_{t} \mid z_{1: t-1}\right)=\sum_{x_{t-1}} P\left(x_{t} \mid x_{t-1}\right) p\left(x_{t-1} \mid z_{1: t-1}\right)$

where $P\left(x_{t} \mid x_{t-1}\right)$ is the probability allotment for state conversion. The condition consequential allocations are anticipated in every occasion by continual submission of prophecy (2) and renew (1). The probability transmits the pragmatic outline $z_{t}$ in the present image to the unidentified pretense, $x_{t}$. A normal distribution as the probability utility is given by (3):

$p\left(z_{t} \mid x_{t}\right) \sim \frac{1}{\sqrt{2 \pi \sigma^{2}}} \exp \left(-\left(d\left(s_{i}, s_{m}\left(x_{t}\right)\right)^{2} / 2 \sigma^{2}\right)\right)$

where $d\left(s_{i}, s_{m}\right)$ is outline aloofness amid the shape practical in the image $s_{i}$ in the present framework and the replica outline $S_{m}$ produced from the 3D hand mock-up in pretense $x_{t}$, and the variance $\sigma^{2}$ is certain experimentally. The preference of the aloofness for evaluating two shapes is vital, as it entails elevated discriminative control as well as speedy estimation. An uncomplicated alternative is the XOR expanse in a predetermined hurdling window was given by the equation (4):

$d_{X O R}\left(s_{i}, s_{m}\right)=1 /|\omega| \sum_{k \in \omega} 1-\left(\delta s_{i}(k), s_{m}(k)\right)$

where $\delta$ is the Kronecker delta utility (1-XOR). Though, this outlay weigh distinctly locked to the shape identical to those sealed to the framework of the outline, it is responsive to discrepancy of hand outline. In turn to highlight structural differentiation involving the shapes, the 'core weighted' XOR aloofness is definite as:

$$
\begin{aligned}
d_{\omega X O R}\left(s_{i}, s_{m}\right)= & 1|\omega| \sum_{k \in \omega}[1 \\
& \left.-\left(\delta s_{i}(k), s_{m}(k)\right)\right] \hat{d}(k)
\end{aligned}
$$

where the weight

$\hat{d}(k)=D\left(s_{i}\right)(k)+\propto D\left(\bar{s}_{i}\right)(k)$

provides dissimilar credence to diverse kinds of mismatches. $D\left(s_{i}\right)$ is the aloofness change of the image outline $S_{i}$ such as the foreground 1 , background 0 and is zero surrounded by the outline and augments with the detachment from the shape. $\bar{S}_{i}$ is the pixel-wise converse of the outline and its aloofness utility is zero external to the curve and elevated in provinces close to the core region, i.e. the 'shape outline'. The weight $\propto$ is put to 6 in (6), thus extremely chastising the case when summits within the image outline are not sheltered by the mock-up protuberance. One disadvantage of this preference of shape aloofness is that pixels on dissimilar fractions of the form carcass have diverse consequences [5]. This is since the expanse change $D\left(\bar{s}_{i}\right)$ commonly encloses superior standards for outsized corpse fractions such as the trunk, principal to insecurity when guesstimating the pretense of thinner boughs. Consequently, standardize the credence such that every pixel on the outline carcass has the similar credence. This is prepared by separating the accurate expression in (6) by the aloofness connecting the shape and the carcass:

$\hat{d}(k)=D\left(s_{i}\right)+\bar{\propto}\left[\frac{D\left(\bar{s}_{i}\right)}{D\left(\bar{s}_{i}\right)+D\left(S_{s k l}\right)}\right]$

where $S_{s k l}$ and $D\left(S_{s k l}\right)$ are the carcass of the outline contour and its aloofness conversion, correspondingly. The credence of a pixel inside the outline is regularized by isolating the aloofness from the outline shape, $D\left(\bar{s}_{i}\right)$, by the distance amid the outline and the cadaver, $D\left(\bar{s}_{i}\right)+D\left(S_{s k l}\right)$. The outline framework is definite as the crease of the standards in the aloofness malformed representation $D\left(\bar{s}_{i}\right)$ for a hallucination of the weights for the soothed core-weighted XOR remoteness as shown in (7). It reveals the enhancement in stoutness over other expense effectiveness by means of utilized color supported backdrop subtraction where the normalization color canons by their strength and replica the pixel-wise distributions with Gaussian pdfs are employed [8]. In every framework, the outline is perceived as the position of pixel with a Mahalanobis aloofness larger than a threshold [6]. A first order progression model is used as a vibrant reproduction, which is candid to assess and demonstrates good flexibility to unidentified motion as publicized underneath:

$p\left(x_{t} \mid x_{t-1}\right) \sim N\left(x_{t-1}, \Sigma\right)$

where $\sum$ is a transverse covariance matrix and the variance $\sigma_{j}$ for every hand splinter group $\mathrm{j}$ is dogged from the existing proposition information, because a lone camera, selfocclusion arises regularly. In such scenario, steady trailing of the occluded hand trailing is tricky since the straightforward vibrant mock-up as illustrated in equation (8) be unsuccessful at some point in occlusion. Though, the scheme is competent of guesstimating the incident of self-occlusions by means of the 3D hand representation whose protuberance is not 
dispersed to any pixel [7]. The variance in the vibrant form of the occluded hand pointer is progressively augmented in (9):

$$
\begin{aligned}
\sum=\operatorname{diag}\left(\sigma_{j}^{2}\right), \sigma_{j}^{\prime} & \\
& =\left\{\begin{array}{c}
\sigma_{j} \text { if part } j \text { is visible } \\
m \sigma_{j} \text { if part } j \text { is occluded }
\end{array}\right.
\end{aligned}
$$

where $m>1$ is a constraint for escalating the standard deviation of an occluded hand. The value of $\mathrm{m}$ is assigned to be 5 in the try outs. While the occluded hand pointers are pragmatic once more, these hand pointers have large variance and the surveillance is confident.

\section{HAND TRAILING AND SIGNAL APPRECIATION}

The trailing fallout of the proposed scheme based on 3D motion capturing Xography on several ten second trail strings poised of dissimilar kinds of hand motions are appraised. The anticipated algorithm can heartily recognize exigent hand configurations with quick shift and crucial self-occlusion. With incomplete position, legitimate statistics, the precision by calculating reprojection error and jitter by figuring the detour from the neighboring standard $\mathrm{d}\left(\mathrm{q}_{\mathrm{i}},|\mathrm{S}| \mathrm{P}_{\mathrm{j}} \in \mathrm{Sq}_{\mathrm{i}+\mathrm{j}}\right)$ of a six-frame window $S=\{-2, ., 2\}$ are assessed to analyze the covariance of $\mathrm{c}$ and $\mathrm{q}_{\mathrm{p}}$ concerning these neighboring standards,

$$
\begin{aligned}
& R=1 / \mathrm{N} \sum_{\mathrm{i}=1}^{\mathrm{N}}\left(\mathrm{c}^{\mathrm{i}}-\mathrm{f}\left(\overline{\mathrm{q}}_{\mathrm{p}}^{\mathrm{i}}\right)\right)\left(\mathrm{c}^{\mathrm{i}}-\mathrm{f}\left(\overline{\mathrm{q}}_{\mathrm{p}}^{\mathrm{i}}\right)\right)^{\mathrm{T}} \\
& Q=1 / \mathrm{N} \sum_{i=1}^{N}\left(\mathrm{q}_{\mathrm{p}}^{\mathrm{i}}-\overline{\mathrm{q}}_{\mathrm{p}}^{\mathrm{i}}\right)\left(\mathrm{q}_{\mathrm{p}}^{\mathrm{i}}-\overline{\mathrm{q}}_{\mathrm{p}}^{\mathrm{i}}\right)^{\mathrm{T}}
\end{aligned}
$$

As anticipated, inverse kinematics decreases reprojection error by imposing a lay down of matching projection constrictions. Though, there is momentous jitter by the side of the ocular axis. By daunting the sequential smoothness tenure, this jitter is greatly castigated and the trailed motion is much smoother. Although sequential smoothing diminishes jitter, it does not purge rational faults down the ocular axis. To envisage these faults, a second camera with its ocular axis vertical to the first camera can be positioned. In corroboration video succession, it was frequently pragmatic that the overall paraphrase faults are flanked by 5 to $10 \mathrm{~cm}$. When the hand pointer is secluded from the camera, the fault might be as high as $15 \mathrm{~cm}$. To recompense for this rational fault in the proposed system by affording visual pointer to the user with a practical hand pointer was cracked. Though the system is likely to be low price, the accumulation of any second camera in harmony to applications accurateness, trims down the profundity in distinctness and might be a better substitution with increased economy. Solitary stranded recital of the proposed system is roughly $140 \mathrm{~ms}$ per frame opening athwart the subsequent errands: $70 \mathrm{~ms}$ for image dispensation, $50 \mathrm{~ms}$ for adjacent neighbor lookup, and $30 \mathrm{~ms}$ for inverse kinematics [9]. Pipelining on a multi-core processor to increase efficacy is used. All the interactive demonstrations of the proposed method sprint at $9 \mathrm{~Hz}$ with a latency of $150 \mathrm{~ms}$ on a $2.4 \mathrm{GHz}$ Intel Core i5 CPU.

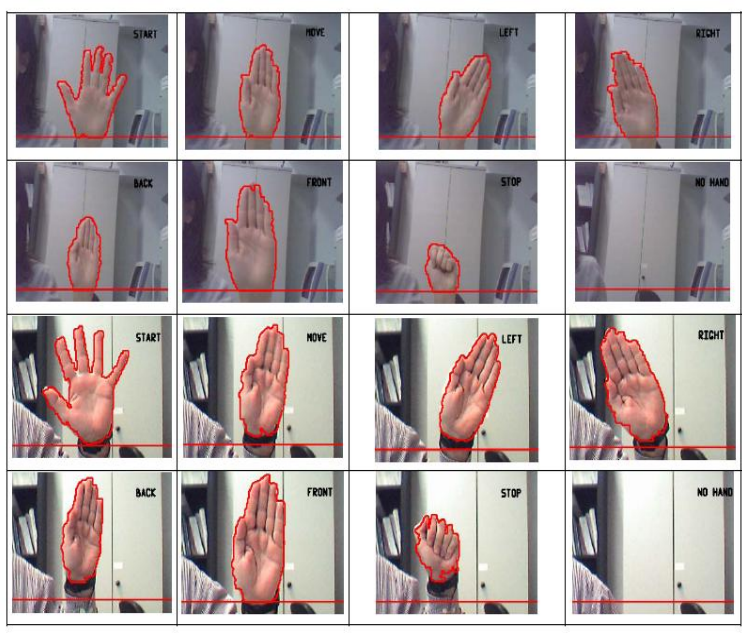

Fig. 1 Hand Trailing and Signal Appreciation

Subsequently, if understood that at time t, $M$ dribbles have been professed, where each dribble commune to any situate of its associated color pixels, the trailing procedure must situate the relative amid the hand premise and the surveillance at occasions. Consecutively to handle with this predicament, a ballpark figure was definite to the expanse from the pixel, $x=(x, y) r$ to the theory. Principally, the pixel harmonics are regularized by means of a yardstick $2 \mathrm{D}$ alternation matrix about the derivation, $\alpha$ is the alternation angle, with $\vec{n}=$ $\left(n_{x}, n_{y}\right)$ the regularize pixel harmonics. In that case, the crossing point if found $\vec{c}=\left(c_{x}, c_{y}\right)$ amid the hand theory ellipse and the standardized pixel as $c_{x}=\omega \cdot \cos \theta$ and $c_{y}=h \cdot \sin \theta$, Where $\vartheta$ is the slant between the regularized pixel and the hand theory. Ultimately, the expanse from a pixel to the hand theory is $\mathrm{d}(\vec{x}, h)=\|\vec{n}-\hat{c}\|$. This expanse can be observed as the estimation of the detachment from a point in the $2 \mathrm{D}$ liberty to a regularized ellipse. Regularized means centered in starting point and not swiveled. From the expanse description, it twirls out that its significance is equivalent or beneath 0 as shown in Fig 1 .

\section{EXPERIMENT AND APPLICATION}

A color camera with an image resolution of $1366 \times 786$ pixels, and down sampling of $683 \times 393$ pixels was betrothed. The pretenses are composed by Xography based 3D motion capturing method where the pretenses hoarded are those that deviate not less than 6 degrees in any mutual view point from other pretenses. The entire amount of pretense is 1,01,438. For precise hand trailing it is useful for the 3D signal appreciation mock-up to have an analogous shape to the present area under discussion. Prior to trailing, shapes and inverse kinematics for every hand trailing replica are appraised. Every shape is encumbered into RAM before trailing, entailing roughly $500 \mathrm{MB}$. One of the kinematic is preferred based on the person's hand size to be tracked. In the proposed 3D motion capturing Xography method, a reliable backdrop is exercised for constant shape mining; though this is not obligatory if the backdrop inference is adequately stout. The method is competent of trailing such an emaciated motion based on inverse kinematics. The computational time fluctuates based on the amount of probability estimations. The standard processing time per frame is $126 \mathrm{~ms}$ utilizing a highend PC as shown in Table 1. 
Table 1. Computation Time in [ms/frame]

\begin{tabular}{|l|c|c|c|}
\hline \multirow{2}{*}{} & \multicolumn{3}{|c|}{ PC } \\
\cline { 2 - 4 } & Min & Max & Avg \\
\hline Capture & 21 & 25 & 22 \\
\hline Pose estimate & 22 & 236 & 104 \\
\hline Total & 43 & 261 & 126 \\
\hline
\end{tabular}

\section{CONCLUSION}

In this manuscript, pioneered a hand trailing and gesture appreciation for user input device sensible of a solitary camera based on 3D motion capturing Xography for video games. Xograph based 3D motion capture performance for several canonical 3D exploitation and pretense appreciation errand was time-honored. Distinctively, this procedure levels the progress of constructive contribution for relatively a lot of interactive appliances. There are numerous probable expansions to the anticipated method. 3D motion capturing Xography can be supported with more cameras for further accuracy or bimanual effort if the hands do not occlude intertwines. 3D motion capturing Xography can be amalgamated with multi-touch influence campaigns to support specific 2D touch contribution. Struts such as a clicker or trigger to alleviate assortment errands can be premeditated. The camera calibration procedure to sustain instant manipulation in a wider series of neighborhoods can be sophisticated. 3D motion capturing Xography in computer animatronics, 3D illustration, novel desktop user interfaces and unconventional perceptive games can be envisioned. Existing procedures for hand pointer trailing to apprehend gesture appreciation to appliances such as implicit surgery to virtual congregation can be influential. The objective of this paper is first and foremost to convey a vigorous and economical user-input contrivance to make available a hand trailing technique with the intention that other researchers may expand ingenious applications of them possess was deliberated.

\section{REFERENCES}

[1] Vanessa. 2014. The Xograph: An Investigation of Parallax Panoramagrams and earlier Autostereoscopic Methods. Film, Photographic and Collections Management.

[2] P.S.Jagadeesh Kumar. 2016. Bespoked Xography for Pointer Trailing and Signal Appreciation of Computer Games. 18th International Conference on Computer Vision Theory and Applications, Tokyo, Japan. World Academy of Science, Engineering and Technology.

[3] Gareth Edwards, Haijiang Li and Bin Wang. 2015. BIM based collaborative and interactive design using computer game engine for general end-users. Visualization in Engg. Springer Open Journal.

[4] Ben Medler, Brian Magerko. 2011. Analytics of Play: Using Information Visualization and Gameplay Practices for Visualizing Video Game Data. Parsons Journal of Information Mapping, New York.

[5] Kathleen Tuite1, Noah Snavely. 2010. Reconstructing the World in 3D: Bringing Games with a Purpose Outdoors. ACM.

[6] P.S.Jagadeesh Kumar, N.Venkateswaran, R.Vijaya Prakash, Pittala Manasa. 2016. Pragmatic Delineation of Multifaceted Vibrant Scheme for Imprudent Animatronics. International Journal of Electrical and Computer Engineering.

[7] Mark Guttenbrunner, Christoph Becker, Andreas Rauber. 2010. Keeping the Game Alive: Evaluating Strategies for the preservation of console video games. The International Journal of Digital Curation.

[8] P.S.Jagadeesh Kumar, S.Meenakshmi Sundaram. 2015. Understanding the Contrive Confront of 3D Video Game Augmentation and Contraption. International Journal of Computer Science and Information Security, USA.

[9] N.Venkateswaran, P.S.Jagadeesh Kumar and Leo L M I. 2016. Tangible Pointer Trailing and Signal Appreciation of Electronic Video Gaming. Communications on Applied Electronics. 